\title{
The Components of Economic Freedom, Income and Growth: An Empirical Analysis
}

\author{
- RAPHAEL B. CORBI*
}

\section{RESUMO}

Este artigo procura trazer um melhor entendimento da relação entre crescimento econômico e os fatores que constituem os elementos da liberdade econômica. Os dois principais objetivos do artigo são: (I) baseados no modelo neoclássico aumentado de crescimento, testar quais dos elementos componentes da liberdade econômica demonstram ter uma relação estatisticamente significante com crescimento; e (2) estabelecer para que lado ocorre a principal direção de causalidade entre liberdade econômica e crescimento. Finalmente, nós identificamos direções desejáveis para novas pesquisas e implicações para política econômica.

\section{PALAVRAS-CHAVE}

liberdade econômica, crescimento, Granger, causalidade

\begin{abstract}
This paper seeks to bring a better understanding of the relationship between economic growth and the disaggregated factors which constitute the elements of economic freedom. The two main objectives of this paper are to: (I) based on the Solow augmented growth model, test which of the elements of economic freedom demonstrate a statistically significant relationship to economic growth; and (2) establish which way the main causality direction between economic freedom and growth runs from. Finally, we identify desirable directions for further research and policy implications.
\end{abstract}

\section{KEY WORDS}

economic freedom, growth, Granger, causality

\section{JEL CLASSIFICATION}

O4

* Departamento de Economia - FEA/USP. Endereço para contato: Rua Carlos del Prete, n. 265, ap. I0I. Centro - São Bernardo - SP. CEP: 097I0-230. E-mail: raphaelcorbi@hotmail.com.

(Recebido em julho de 2005. Aceito para publicação em setembro de 2006). 
The relationship between economic freedom and growth has been studied and debated by economists since Adam Smith. It has been generally argued that free economies will be more productive. Economic freedom is considered to be the ultimate incentive for the optimal use of scarce resources, by creating a favorable environment for healthy competitiveness and stimulating human creativity. In a free private market, individuals have the freedom to choose what to consume, to produce, and to give. The invisible hand leads free economic agents to pursue their own interests and voluntarily cooperate with others. (Smith, 1776).

All the beliefs discussed above sound very reasonable and seem to be widely accepted among economists. But the empirical evidence to support such convictions has been largely ambiguous and incomplete until very recently. The debate on this question has evolved to more unambiguous grounds only recently, with the development of sophisticated standard measures of economic freedom for a large number of economies. Although many recent works have shown a consistent positive correlation between economic growth and overall economic freedom indices, the literature still lacks enough evidence on which aspects of economic freedom mostly contribute to economic expansion. The present work seeks to shed more light on this question in order to identify which elements of economic freedom are more relevant.

This paper is divided into 4 sections. Section 1 reviews the literature. Section 2 discusses economic freedom measurement and describes the data. In section 3, we present the augmented Solow growth model which is the theoretical background behind our subsequent analysis on the impact of the disaggregated factors which constitute the elements of economic freedom on growth. Moreover, we perform a Granger-causality test to properly assess where the main causality direction runs from. Finally, we conclude.

\section{LITERATURE}

Economists believe that economic freedom will enhance growth and development through many different ways. Gwartney and Lawson (2004) highlight the importance of three of them: competition, entrepreneurship and investment. Competitive markets will allow free entry for new business firms. The only firms that will survive are the ones which are able to provide costumers with quality products at low costs. As a result of this system, we can expect a higher level of output using a reduced and optimal amount of input. Entrepreneurial discovery is highly stimulated in free economies, creating a continuous process of experimentation and discovery which is a 
powerful force for economic progress. Finally, economic theory indicates that private investment will tend to flow toward economic environments that are more attractive for productive activities. Free economies will attract more investment, which in turn will promote economic growth. On the other hand, high taxes, excessive regulation, biased enforcement of contracts, lack of legal recourse, insecure property rights, and monetary instability will deter both investment and growth.

Research has shown a positive relationship between economic freedom and growth. As one would expect, the better property rights are protected, the more of their incomes individuals get to keep, and the fewer restrictions placed on entrepreneurial activity, the more growth-producing economic activity takes place. Indeed, using both EFN and HF indices, Norton (1998) finds that better specified property rights are associated with higher levels of human development and have a strong influence on the well-being of the world's most impoverished people. Using the Economic Freedom index produced by the Frasier Institute (also used by the present study), Ayal and Karras (1998) show that aggregate economic freedom appears to enhance growth both via increasing total factor productivity and via enhancing capital accumulation. On the other hand, de Haan and Siermann (1998) find that the link between freedom and growth depends upon the freedom measure used, and that capital accumulation is not significantly related to economic freedom. Easton and Walker (1997) show that improvements on economic freedom have impact of great magnitude on steady-state income levels. Other interesting finding is that greater economic freedom enhances quality of life both across nations and increases the improvements in quality of life over time. (Esposto and Zaleski, 1999).

Some economists have also theorized that the more economic growth there is, the less equal the income distribution is - suggesting a conflict between growth and equality. Researchers traditionally assume greater equality is achieved through growth-reducing taxes and regulations, and the redistribution of income after it is produced. The economist Niclas Berggren (1999) looked at the problem differently, by investigating the relationship between economic freedom and equality. Berggren, comparing the EFN Index to recent data on income distribution in 102 countries, he concluded that to achieve economic growth and equality, policy-makers should steadily increase economic freedom over time. This result was not only true for developed, higher-income countries, but was especially true for low-income, less-developed countries. Even, the higher level of equality came primarily through faster growth in the gross incomes of poor people than in the incomes of rich people, on average. 


\section{ECONOMIC FREEDOM AND DATA DESCRIPTION}

In the early 1990's, a lot of effort was put into the attempt to design a reliable measure of economic freedom. Many leading economic scholars including Nobel laureates Milton Friedman, Douglass North and Gary Becker, turned to this matter and made absolutely essential contributions to the area. All this hard work culminated shortly after with the establishment of several objective indices of economic freedom, highlighting the Economic Freedom Network Index (EFNI), produced by the Frasier Institute, and the Heritage Foundation's Index (HFI). Both the EFNI and HFI attempt to obtain an overall economic freedom ranking for each country during a particular year based on raw scores on a variety of factors relevant to economic freedom. They follow a similar procedure that contains the following elements: defining economic freedom; selecting component variables; rating component variables; combining component ratings into the final overall rankings of economic freedom.

There have been serious attempts to compare both indices available in the recent literature. The two indices share some similarities and some differences in their methods of measuring economic freedom. Wu and Davis (2005) investigated whether the different methods would lead to differences in their final ratings of economic freedom. To statistically compare the two indices, they compile a data set which includes 238 country-years in 1995 and 1999. Results clearly demonstrate a pattern in which observations classified as economically freer in the HFI are also classified as economically freer in the EFNI. The numbers indicate that both indices are highly correlated. They further explore the relationship between the two indices by testing the null hypothesis of independence between the two categorical variables. The Pearson chi-squared statistic is 218.43 which yields a P-value less than 0.0001 , and the likelihood-ratio chi-squared statistic is 197.49 with a P-value less than 0.0001 . Authors conclude that there is very strong evidence of association between these two measures.

The two indices differ significantly in ways in which they rate on components of economic freedom. The HFI uses a five-level grading scale to determine scores for each factor based on information collected on pertinent factor variables. However, not all factor variables are individually graded. Therefore, raw data on factor variables are combined into factor grades in a relatively subjective way. There are no explicit formulas for summarizing information on factor variables into factor grades. The EFNI directly assigns scores to all component variables on a 0 -to-10 scale. For continuous component variables, this index applies explicit and fixed formulas to convert original data on component variables into scores. For categorical component variables, subjective judgments are applied to obtain scores. Areas of economic freedom in the EFNI are rated solely on the scores of pertinent component variables. In comparison, 
scores on the factors in the HFI seem to be obtained in a more subjective way than scores on areas in the EFNI.

Another point in which the two indices differ is their weighting schemes for combining component ratings into their final overall rankings of economic freedom. According to Wu and Davis (1999), while the HFI simply weights factor ratings equally, the EFNI uses principle component analysis to construct weights for each component variables in calculating the final scores of economic freedom in order to allow one to obtain a measure of economic freedom which is statistically objective in the sense that the final ratings of economic freedom are directly derived from the data of component variables. The method suits well for the exercise of measuring economic freedom since the final overall scores of economic freedom are derived from components that are assumed to reflect some aspects of the concept of economic freedom. The weights are based on the principal component that explains the maximum variations in the original data of component variables among all standardized linear combinations of the original data.

Given all the information cited above, we believe that the EFNI is the best index available and that it provides a reliable measure of cross-country differences in economic freedom, so we decide to use this index in our present work. However, measuring economic freedom is still a work in progress and constructing an ideal objective measure does not seem to be a very realistic task. Some components of economic freedom have proven particularly difficult to measure because it was practically impossible to obtain the required data for a large number of countries or, in other cases, potential variables were omitted because their nature virtually precluded objective measurement. It is particularly difficult to quantify the impact of regulation objectively. Nonetheless, restrictive regulations can exert an important influence on the degree of economic freedom present in a country.

The concept of Economic Freedom adopted here is consequently the one defined by the Economic Freedom of the World Network, as it is given in its 2004 Annual Report. We consider as the key ingredients of economic freedom the following: personal choice, voluntary exchange, freedom to compete, and protection of person and property. Institutions and policies are consistent with economic freedom when they provide an infrastructure for voluntary exchange and protect individuals and their property from aggressors seeking to use violence, coercion, and fraud to seize things that do not belong to them. Governments promote economic freedom when they provide a legal structure and a law enforcement system that protect the property rights of owners and enforce contracts in an effective manner. They also enhance economic freedom when they facilitate access to sound money. (Gwartney, James and Lawson, 2004). On the other hand, governments concerned with economic freedom should refrain 
from actions that interfere with it. For instance when taxes, government expenditures, and regulations are substitutes for personal choice, voluntary exchange, and market coordination, the result is a reduction on economic freedom. It may also be damaged by restrictions that limit entry into occupations and business activities.

All the findings discussed in section $\mathrm{l}$ are result of serious efforts from investigators trying to better understand how economic freedom contributes to economic development. We cannot deny the importance and all the insight that all these studies have given us all. But most of this work has been done using only the indices available, ignoring the different components of economic freedom. The methodology adopted for calculating the EFN index condenses too much diverse information into one single summary number and that could lead one to jump to incorrect conclusions. An uninformed investigator that treated economic freedom as a one-dimension homogenous piece of information when analyzing cross-country data could make imprecise affirmatives concerning the relationship between economic freedom and income.

On Table 1, we find how the EFN index is compound of five different areas (size of government, legal structure and security of property rights, access to sound money, freedom to trade internationally and regulation of markets) and 21 sub-areas. We strongly believe that these areas and sub-areas have very distinguished nature and there is nothing we can think of that could make us believe that they somehow should present a combined behavior, that is, there is no reason for us to assume that these factors should be treated jointly as an aggregate. Suppose, for instance, we were investigating the relationship between economic freedom as a whole and growth and we find that it is positive and significant. Is it sensible to just conclude that countries with small-sized governments should grow faster? Is it not also possible that economic growth induces reductions on the size of governments? Even, can it not be the case in which growth potential increases with low government consumption but lowers with low public investment? These are just a sample of all the problems and doubts that might arise when leading such kind of study. 
TABLE 1 - THE AREAS AND SUB-AREAS OF THE EFN INDEX

\section{1 - Size of Government: Expenditures, Taxes, and Enterprises}

A Government consumption spending as a percentage of total consumption

B Transfers and subsidies as a percentage of GDP

C Government enterprises and investment as a percentage of total investment

D Top marginal tax rate (and income threshold at which it applies)

\section{2 - Legal Structure and Security of Property Rights}
A Judicial independence - the judiciary is independent and not subject to interference by the government or parties in disputes
B Impartial courts-a trusted legal framework exists for private businesses to challenge the legality of government actions or regulation
C Protection of intellectual property
D Military interference in rule of law and the political process
E Integrity of the legal system

\section{3 - Access to Sound Money}

A Average annual growth of the money supply minus real GDP in the last five years

B Standard inflation variability in the last five years

C Recent inflation rate

D Freedom to own foreign currency bank accounts domestically and abroad
4 - Freedom to Trade Internationally
A Taxes on international trade
B Regulatory trade barriers
C Actual size of trade sector compared to expected size
D Difference between official exchange rate and black-market rate
E International capital market controls

\section{5 - Regulation of Credit, Labor, and Business}
A Credit market regulations
B Labor market regulations
C Business regulations

Source: Gwartney, James and Lawson, Robert (2004). 


\section{The Data}

Our data on economic freedom is available for 114 countries and for the years 1970, 1975, 1980, 1985, 1990, 1995 and 2000. Table 2 presents summary economic freedom ratings and its five components (and rankings) for the year 2000 as an illustration. The higher the rating, the closer a country is to a truly free economic environment. Hong Kong, United States and Singapore occupy the top three positions on the overall index ranking. The other nations in the top 10 are New Zealand, Switzerland, United Kingdom, Canada, Ireland, Australia and Netherlands. All countries at the bottom of the list are notably African nations: the Republic of Congo, Guinea-Bissau, Algeria, Zimbabwe, Myanmar, and, in last place, the Democratic Republic of Congo.

A number of interesting patterns emerge from an analysis of these data. The highincome industrial economies generally rank quite high for Legal Structure and Security of Property Rights (Area 2), Access to Sound Money (Area 3), Freedom to Trade Internationally (Area 4) and Regulation of Credit, Labor, and Business (Area 5). Their ratings were lower, however, for Size of Government: Expenditures, Taxes, and Enterprises (Area 1). This was particularly true for western European countries. On the other hand, a number of developing nations show the opposite pattern. Bolivia makes an interesting case study. It shows that reasonably sized government is not enough to reap the benefits of economic freedom. The institutions of economic freedom, such as the rule of law and property rights, as well as sound money, trade openness, and sensible regulation are required. Bolivia was ranked $19^{\text {th }}$ in Size of Government: Expenditures, Taxes, and Enterprises and $31^{\text {st }}$ for Access to Sound Money. However, it scored poorly in all the other categories, especially Legal Structure and Security of Property Rights, where it placed $113^{\text {th }}$. Despite high rankings in a couple of areas, Bolivia's overall ranking is only $45^{\text {th }}$. Weakness in the rule of law and property rights is particularly pronounced in sub-Sahara Africa, among Islamic nations, and for several nations that were part of the former Soviet bloc. However, many Latin American and Southeast Asian nations also score poorly for rule of law and property rights. The nations that rank poorly in this category also tend to score poorly in the trade and regulation categories, even though several of these nations have reasonably sized governments and sound money. 
TABLE 2 - AREA ECONOMIC FREEDOM RATINGS (AND RANKINGS), 2000

\begin{tabular}{|c|c|c|c|c|c|c|c|c|c|c|c|c|}
\hline \multirow{3}{*}{$\begin{array}{l}\text { COUNTRY } \\
\text { Hong Kong }\end{array}$} & \multirow{2}{*}{\multicolumn{2}{|c|}{ INDEX }} & \multirow{2}{*}{\multicolumn{2}{|c|}{$\begin{array}{l}\text { AREA } 1 \\
\text { Size of Govern- } \\
\text { ment: Expen- } \\
\text { ditures, Taxes, } \\
\text { and Enterprises }\end{array}$}} & \multirow{2}{*}{\multicolumn{2}{|c|}{$\begin{array}{c}\text { AREA 2 } \\
\text { Legal Structure } \\
\text { \& Security } \\
\text { of Property } \\
\text { Rights }\end{array}$}} & \multirow{2}{*}{\multicolumn{2}{|c|}{$\begin{array}{l}\text { AREA } 3 \\
\text { Access to } \\
\text { Sound } \\
\text { Money }\end{array}$}} & \multirow{2}{*}{\multicolumn{2}{|c|}{$\begin{array}{l}\text { AREA 4 } \\
\text { Freedom to } \\
\text { Trade } \\
\text { Internationally }\end{array}$}} & \multirow{2}{*}{\multicolumn{2}{|c|}{$\begin{array}{c}\text { AREA 5 } \\
\text { Regulation of } \\
\text { Credit, Labor, } \\
\text { and Business }\end{array}$}} \\
\hline & & & & & & & & & & & & \\
\hline & 8,7 & (1) & 9,2 & (1) & 7,2 & (26) & 9,1 & (40) & 9,8 & (1) & 8,4 & (1) \\
\hline United States & 8,6 & (2) & 7,6 & (15) & 9,2 & (9) & 9,8 & (3) & 8,1 & (25) & 8,2 & (2) \\
\hline Singapore & 8,5 & (3) & 8,0 & (8) & 8,5 & (17) & 9,5 & (23) & 9,3 & (2) & 7,3 & (8) \\
\hline New Zealand & 8,4 & (4) & 6,7 & (35) & 9,1 & (11) & 9,6 & (17) & 8,5 & (11) & 7,9 & (4) \\
\hline Switzerland & 8,3 & (5) & 7,0 & (28) & 9,3 & (7) & 9,7 & (7) & 8,5 & (10) & 7,3 & (9) \\
\hline Unit. Kingdom & 8,3 & (5) & 6,2 & (51) & 9,3 & (6) & 9,3 & (29) & 8,4 & (12) & 8,1 & (3) \\
\hline Canada & 8,1 & (7) & 6,0 & (59) & 9,3 & (8) & 9,5 & (22) & 8,3 & (16) & 7,6 & (5) \\
\hline Ireland & 8,1 & (7) & 6,1 & (54) & 9,0 & (14) & 9,4 & (25) & 9,1 & (3) & 7,0 & (14) \\
\hline Australia & 8,0 & (9) & 6,2 & (53) & 9,5 & (3) & 9,4 & (28) & 7,8 & (33) & 7,2 & (10) \\
\hline Netherlands & 8,0 & (9) & 4,5 & (97) & 9,6 & (1) & 9,6 & (15) & 9,0 & (5) & 7,6 & (6) \\
\hline Luxembourg & 7,8 & (11) & 4,5 & (95) & 8,6 & (16) & 9,7 & (4) & 9,0 & (4) & 6,9 & (20) \\
\hline Denmark & 7,7 & (12) & 3,6 & (112) & 9,5 & (2) & 9,7 & (6) & 8,3 & (18) & 7,2 & (11) \\
\hline Finland & 7,7 & (12) & 4,1 & (101) & 9,5 & (4) & 9,5 & (18) & 8,3 & (14) & 7,1 & (13) \\
\hline Iceland & 7,7 & (12) & 5,9 & (62) & 9,0 & (12) & 9,2 & (35) & 6,9 & (66) & 7,6 & (7) \\
\hline Germany & 7,6 & (15) & 4,5 & (96) & 9,1 & (10) & 9,6 & (11) & 8,7 & (8) & 6,1 & (51) \\
\hline Unit. Arab Em. & 7,6 & (15) & 7,6 & (13) & 6,6 & (39) & 9,1 & (36) & 8,2 & (21) & 6,5 & (39) \\
\hline Austria & 7,5 & (17) & 3,4 & (117) & 9,3 & (5) & 9,6 & (9) & 8,6 & (9) & 6,5 & (37) \\
\hline Belgium & 7,5 & (17) & 3,5 & (116) & 8,3 & (18) & 9,6 & (12) & 9,0 & (6) & 7,0 & (18) \\
\hline Chile & 7,5 & (17) & 7,1 & (22) & 6,5 & (42) & 9,3 & (32) & 7,5 & (43) & 7,0 & (17) \\
\hline Oman & 7,4 & (20) & 5,8 & (67) & 6,9 & (32) & 9,8 & (2) & 7,8 & (32) & 6,8 & (24) \\
\hline Spain & 7,4 & (20) & 4,6 & (92) & 7,5 & (25) & 9,4 & (26) & 8,3 & (17) & 6,9 & (19) \\
\hline Sweden & 7,4 & (20) & 3,0 & (119) & 9,0 & (13) & 9,8 & (1) & 8,3 & (15) & 6,8 & (23) \\
\hline Costa Rica & 7,3 & (23) & 7,1 & (21) & 6,9 & (35) & 7,9 & (64) & 8,0 & (26) & 6,6 & (33) \\
\hline El Salvador & 7,3 & (23) & 8,4 & (5) & 4,5 & (86) & 9,4 & (27) & 7,5 & (42) & 6,4 & (43) \\
\hline Japan & 7,3 & (23) & 5,3 & (76) & 8,2 & (19) & 9,6 & (10) & 6,8 & (68) & 6,6 & (32) \\
\hline Mauritius & 7,3 & (23) & 6,9 & (29) & 6,9 & (31) & 9,6 & (13) & 6,8 & (70) & 6,1 & (53) \\
\hline Portugal & 7,3 & (23) & 5,1 & (83) & 7,6 & (24) & 9,5 & (20) & 8,1 & (24) & 6,4 & (42) \\
\hline Argentina & 7,2 & (28) & 7,8 & (10) & 5,4 & (68) & 9,7 & (5) & 6,4 & (81) & 6,7 & (28) \\
\hline Bahrain & 7,2 & (28) & 6,8 & (32) & 5,9 & (59) & 9,1 & (38) & 7,7 & (36) & 6,7 & (30) \\
\hline Botswana & 7,2 & (28) & 5,3 & (80) & 6,8 & (36) & 9,1 & (39) & 7,8 & (31) & 7,0 & (15) \\
\hline Norway & 7,2 & (28) & 3,7 & (110) & 8,8 & (15) & 9,0 & (41) & 7,6 & (41) & 6,7 & (31) \\
\hline Taiwan & 7,2 & (28) & 6,2 & $(50)^{\prime}$ & 6,1 & (50) & 9,5 & (21) & 8,1 & (23) & 6,1 & (56) \\
\hline Estonia & 7,1 & (33) & 5,4 & (75) & 6,0 & (51) & 8,6 & (48) & 8,8 & (7) & 6,6 & (35) \\
\hline Italy & 7,1 & (33) & 4,6 & (94) & 7,7 & (23) & 9,5 & (24) & 8,2 & (19) & 5,5 & (82) \\
\hline Panama & 7,1 & (33) & 7,3 & (20) & 5,2 & (72) & 9,6 & (14) & 7,1 & (59) & 6,5 & (38) \\
\hline Philippines & 7,1 & (33) & 7,6 & (12) & 4,6 & (81) & 9,2 & (34) & 7,6 & (37) & 6,4 & (40) \\
\hline France & 7,0 & (37) & 2,3 & (123) & 8,1 & (21) & 9,6 & (16) & 8,2 & (20) & 6,8 & (22) \\
\hline Jamaica & 7,0 & (37) & 7,5 & (16) & 5,2 & (74) & 8,7 & (46) & 7,2 & (56) & 6,4 & (45) \\
\hline Jordan & 7,0 & (37) & 5,0 & (85) & 7,2 & (27) & 9,7 & (8) & 7,0 & (64) & 6,2 & (47) \\
\hline Trinid \& Tob. & 7,0 & (37) & 5,9 & (65) & 5,9 & (56) & 9,1 & (37) & 6,7 & (73) & 7,2 & (12) \\
\hline Greece & 6,9 & (41) & 6,4 & (47) & 5,7 & (63) & 9,3 & (30) & 7,9 & (29) & 5,4 & (87) \\
\hline
\end{tabular}

(continues) 


\begin{tabular}{|c|c|c|c|c|c|c|c|c|c|c|c|c|}
\hline \multirow[t]{2}{*}{$\begin{array}{l}\text { COUNTRY } \\
\text { Peru }\end{array}$} & \multicolumn{2}{|c|}{ INDEX } & \multicolumn{2}{|c|}{$\begin{array}{c}\text { AREA } 1 \\
\text { Size of Govern- } \\
\text { ment: Expen- } \\
\text { ditures, Taxes, } \\
\text { and Enterprises }\end{array}$} & \multicolumn{2}{|c|}{$\begin{array}{c}\text { AREA2 } \\
\text { Legal Structure } \\
\& \text { Security } \\
\text { of Property } \\
\text { Rights }\end{array}$} & \multicolumn{2}{|c|}{$\begin{array}{l}\text { AREA } 3 \\
\text { Access to } \\
\text { Sound } \\
\text { Money }\end{array}$} & \multicolumn{2}{|c|}{$\begin{array}{c}\text { AREA 4 } \\
\text { Freedom to } \\
\text { Trade } \\
\text { Internationally }\end{array}$} & \multicolumn{2}{|c|}{$\begin{array}{c}\text { AREA } 5 \\
\text { Regulation of } \\
\text { Credit, Labor, } \\
\text { and Business }\end{array}$} \\
\hline & 6,9 & (41) & 8,1 & (6) & 3,9 & $(102)$ & 8,8 & (44) & 7,3 & (46) & 6,6 & (34) \\
\hline Malaysia & 6,8 & (43) & 6,7 & (33) & 5,6 & (64) & 8,1 & (57) & 7,5 & (44) & 6,3 & (46) \\
\hline South Africa & 6,8 & (43) & 5,4 & (74) & 6,5 & (40) & 7,9 & (62) & 7,2 & (52) & 6,9 & (21) \\
\hline Bolivia & 6,7 & (45) & 7,5 & (19) & 3,4 & (113) & 9,3 & (31) & 7,3 & (47) & 6,0 & (59) \\
\hline Czech Rep. & 6,7 & (45) & 4,6 & (93) & 6,9 & (34) & 8,1 & (59) & 8,4 & (13) & 5,7 & (67) \\
\hline Egypt & 6,7 & (45) & 6,5 & (39) & 5,9 & (58) & 9,5 & (19) & 6,1 & (91) & 5,2 & (92) \\
\hline Hungary & 6,7 & (45) & 4,8 & (88) & 7,0 & (29) & 7,1 & (77) & 7,7 & (34) & 7,0 & (16) \\
\hline Kuwait & 6,7 & (45) & 6,1 & (55) & 6,9 & (30) & 8,2 & (56) & 7,2 & (51) & 5,1 & (96) \\
\hline South Korea & 6,7 & (45) & 7,1 & (23) & 6,0 & (54) & 8,2 & (54) & 7,1 & (60) & 5,3 & (91) \\
\hline Thailand & 6,7 & (45) & 6,8 & (31) & 6,0 & (53) & 6,6 & (96) & 7,6 & (38) & 6,2 & (48) \\
\hline Uganda & 6,7 & (45) & 6,5 & (42) & 4,6 & (83) & 9,3 & (33) & 7,4 & (45) & 5,6 & (75) \\
\hline Guyana & 6,6 & (53) & 4,0 & (105) & 6,5 & (41) & 7,9 & (63) & 8,1 & (22) & 6,7 & (29) \\
\hline Latvia & 6,6 & (53) & 5,2 & (81) & 5,9 & (55) & 8,6 & (49) & 7,2 & (53) & 6,1 & (55) \\
\hline Nicaragua & 6,6 & (53) & 6,3 & (48) & 4,1 & (100) & 8,7 & (47) & 7,6 & (40) & 6,1 & (52) \\
\hline Uruguay & 6,6 & (53) & 6,0 & (58) & 5,7 & (62) & 8,4 & (51) & 6,8 & (71) & 6,2 & (50) \\
\hline Zambia & 6,6 & (53) & 6,6 & (37) & 5,9 & (60) & 7,2 & (75) & 7,9 & (28) & 5,6 & (77) \\
\hline Bahamas & 6,5 & (58) & 7,5 & (17) & 6,3 & (46) & 6,8 & (87) & 5,0 & (115) & 6,8 & (27) \\
\hline Domen. Rep. & 6,5 & (58) & 8,6 & (3) & 4,5 & (87) & 6,8 & (88) & 6,0 & (93) & 6,5 & (36) \\
\hline Israel & 6,5 & (58) & 2,7 & (122) & 8,0 & (22) & 8,1 & (60) & 7,9 & (30) & 5,9 & (62) \\
\hline Kenya & 6,5 & (58) & 6,6 & (38) & 4,0 & (101) & 8,8 & (43) & 7,1 & (62) & 6,2 & (49) \\
\hline Malta & 6,5 & (58) & 5,9 & (61) & 7,2 & (28) & 7,3 & (71) & 6,4 & (79) & 5,7 & (70) \\
\hline Guatemala & 6,4 & (63) & 9,1 & (2) & 3,4 & (114 & 7,6 & (67) & 6,5 & (74) & 5,5 & (84) \\
\hline Haiti & 6,4 & (63) & 7,8 & (9) & 3,9 & (103) & 8,4 & (52) & 5,8 & (102) & 6,0 & (60) \\
\hline Honduras & 6,4 & (63) & 7,5 & (18) & 3,7 & (109) & 8,2 & (55) & 6,8 & (69) & 5,6 & (72) \\
\hline Lithuania & 6,3 & (66) & 5,6 & (70) & 5,8 & (61) & 7,1 & (76) & 7,3 & (48) & 5,6 & (71) \\
\hline Mexico & 6,3 & (66) & 7,1 & (25) & 4,2 & (97) & 6,9 & (86) & 7,6 & (39) & 5,6 & (74) \\
\hline Namibia & 6,3 & (66) & 4,0 & (104) & 8,1 & (20) & 6,1 & (105) & 6,3 & (82) & 6,8 & (25) \\
\hline Paraguay & 6,3 & (66) & 8,0 & (7) & 3,8 & (105) & 8,7 & (45) & 6,4 & (80) & 4,9 & (104) \\
\hline Poland & 6,3 & (66) & 5,0 & (84) & 6,5 & (43) & 7,5 & (69) & 6,9 & (67) & 5,9 & (64) \\
\hline Slovak Rep & 6,3 & (66) & 3,5 & (115) & 6,3 & (47) & 8,0 & (61) & 7,9 & (27) & 5,8 & (65) \\
\hline Belize & 6,2 & (72) & 5,8 & (68) & 6,2 & (48) & 6,7 & (91) & 5,7 & (105) & 6,8 & (26) \\
\hline Cyprus & 6,2 & (72) & 6,2 & (49) & 6,9 & (33) & 6,9 & (85) & 5,8 & (104) & 5,4 & (89) \\
\hline India & 6,2 & (72) & 6,8 & (30) & 6,0 & (52) & 6,9 & (84) & 5,5 & (109) & 5,8 & (66) \\
\hline Fiji & 6,1 & (75) & 6,1 & (57) & 5,5 & (67) & 7,0 & (80) & 6,2 & (88) & 5,9 & (61) \\
\hline Sri Lanka & 6,1 & (75) & 7,0 & (27) & 4,2 & (99) & 6,7 & (89) & 6,5 & (75) & 6,0 & (57) \\
\hline Tunisia & 6,1 & (75) & 5,3 & (78) & 6,4 & (44) & 7,0 & (79) & 6,1 & (92) & 5,6 & (73) \\
\hline Mali & 6,0 & (78) & 5,7 & (69) & 5,0 & (77) & 8,4 & (50) & 6,4 & (77) & 4,6 & (108) \\
\hline Morocco & 6,0 & (78) & 5,9 & (60) & 6,7 & (37) & 7,0 & (82) & 5,4 & (110) & 5,1 & (98) \\
\hline Brazil & 5,9 & $(80)$ & 5,9 & (66) & 5,4 & (71) & 6,1 & (104) & 5,9 & (96) & 6,0 & (58) \\
\hline Ghana & 5,9 & (80) & 6,1 & (56) & 4,4 & (89) & 5,8 & (107) & 7,3 & (50) & 5,9 & (63) \\
\hline Indonesia & 5,9 & (80) & 7,6 & (14) & 3,4 & (115) & 6,2 & (103) & 7,7 & (35) & 4,6 & (107) \\
\hline Pap. N. Guinea & 5,9 & (80) & 6,5 & (43) & 4,4 & (93) & 6,3 & (102) & 6,3 & (83) & 6,4 & (44) \\
\hline Slovenia & 5,9 & $(80)$ & 2,9 & (120) & 6,4 & (45) & 7,4 & (70) & 7,1 & (61) & 5,7 & (68) \\
\hline China & 5,8 & $(85)$ & 3,8 & (108) & 4,9 & (78) & 8,1 & (58) & 7,2 & (55) & 5,0 & (101) \\
\hline Croatia & 5,8 & (85) & 2,8 & (121) & 6,6 & (38) & 7,8 & (65) & 6,3 & (84) & 5,5 & (81) \\
\hline Madagascar & 5,8 & (85) & 6,5 & (40) & 4,6 & (84) & 7,2 & (74) & 6,2 & (86) & 4,4 & (114) \\
\hline
\end{tabular}




\begin{tabular}{|c|c|c|c|c|c|c|c|c|c|c|c|c|}
\hline \multirow[t]{2}{*}{$\begin{array}{l}\text { COUNTRY } \\
\text { Nepal }\end{array}$} & \multicolumn{2}{|c|}{ INDEX } & \multicolumn{2}{|c|}{$\begin{array}{l}\text { AREA } 1 \\
\text { Size of Govern- } \\
\text { ment: Expen- } \\
\text { ditures, Taxes, } \\
\text { and Enterprises }\end{array}$} & \multicolumn{2}{|c|}{$\begin{array}{c}\text { AREA 2 } \\
\text { Legal Structure } \\
\text { \& Security } \\
\text { of Property } \\
\text { Rights }\end{array}$} & \multicolumn{2}{|c|}{$\begin{array}{l}\text { AREA } 3 \\
\text { Access to } \\
\text { Sound } \\
\text { Money }\end{array}$} & \multicolumn{2}{|c|}{$\begin{array}{l}\text { AREA 4 } \\
\text { Freedom to } \\
\text { Trade } \\
\text { Internationally }\end{array}$} & \multicolumn{2}{|c|}{$\begin{array}{c}\text { AREA } 5 \\
\text { Regulation of } \\
\text { Credit, Labor, } \\
\text { and Business }\end{array}$} \\
\hline & 5,8 & (85) & 5,3 & (77) & 4,8 & (80) & 7,0 & (81) & 6,2 & (87) & 5,6 & (76) \\
\hline Niger & 5,8 & (85) & 5,9 & (63) & 4,4 & (92) & 8,3 & (53) & 6,0 & (94) & 4,6 & (109) \\
\hline Senegal & 5,8 & (85) & 6,7 & (36) & 4,4 & (91) & 7,3 & (72) & 6,3 & (85) & 4,6 & (111) \\
\hline Tanzania & 5,8 & (85) & 4,9 & (87) & 6,1 & (49) & 8,8 & (42) & 5,5 & (108) & 3,7 & (121) \\
\hline Turkey & 5,8 & (85) & 7,1 & (26) & 5,4 & (70) & 3,6 & (116) & 7,2 & (57) & 5,7 & (69) \\
\hline Venezuela & 5,8 & (85) & 7,1 & (24) & 3,7 & (106) & 5,6 & (110) & 7,2 & (54) & 5,2 & (95) \\
\hline Albania & 5,7 & (94) & 6,2 & (52) & 4,6 & (85) & 7,3 & (73) & 5,3 & (112) & 5,0 & (102) \\
\hline Bangladesh & 5,7 & (94) & 7,7 & (11) & 3,5 & (112) & 6,9 & (83) & 5,1 & (113) & 5,5 & (83) \\
\hline Cote d'Ivoire & 5,7 & (94) & 6,5 & (41) & 3,9 & (104) & 6,7 & (90) & 5,9 & (99) & 5,4 & (88) \\
\hline Barbados & 5,6 & (97) & 4,7 & (89) & 5,2 & (73) & 6,6 & (94) & 5,0 & (114) & 6,4 & (41) \\
\hline Benin & 5,6 & (97) & 6,5 & (44) & 4,3 & (94) & 6,6 & (95) & 5,4 & (111) & 5,1 & (100) \\
\hline Iran & 5,6 & (97) & 4,4 & (99) & 5,9 & (57) & 7,8 & (66) & 5,9 & (98) & 3,9 & (118) \\
\hline Cameroon & 5,5 & (100) & 5,5 & (71) & 4,4 & (88) & 6,6 & (97) & 5,8 & (101) & 5,0 & (103) \\
\hline Chad & 5,4 & (101) & 6,5 & (46) & 4,2 & (98) & 6,6 & (92) & 5,8 & (103) & 3,9 & (120) \\
\hline Colombia & 5,4 & (101) & 5,1 & (82) & 3,5 & (110) & 6,3 & (101) & 6,4 & (76) & 5,5 & (80) \\
\hline Ecuador & 5,4 & (101) & 8,4 & (4) & 3,3 & (116) & 3,8 & (114) & 7,1 & (58) & 4,2 & (115) \\
\hline Pakistan & 5,4 & (101) & 6,7 & (34) & 4,6 & (82) & 6,5 & (99) & 4,1 & (120) & 5,2 & (93) \\
\hline Nigeria & 5,3 & (105) & 5,5 & (72) & 3,7 & (107) & 5,3 & (112) & 5,9 & (97) & 6,1 & (54) \\
\hline Bulgaria & 5,1 & (106) & 4,0 & (103) & 5,4 & (69) & 3,2 & (119) & 7,3 & (49) & 5,4 & (86) \\
\hline Burundi & 5,1 & (106) & 6,5 & (45) & 3,3 & (117) & 6,1 & (106) & 4,3 & (119) & 5,5 & (85) \\
\hline Rwanda & 5,1 & (106) & 5,5 & (73) & 2,0 & (123) & 7,6 & (68) & - & (123) & 5,2 & (94) \\
\hline Sierra Lec & 5,1 & (106) & 5,9 & (64) & 3,5 & (111) & 6,5 & (100) & 4,8 & (118) & 4,8 & (106) \\
\hline Togo & 5,1 & (106) & 4,4 & (98) & 3,7 & (108) & 6,5 & (98) & 6,1 & (90) & 4,4 & (113) \\
\hline Gabon & 5,0 & (111) & 3,7 & (111) & 4,3 & (95) & 5,8 & (108) & 5,6 & (107) & 5,5 & (79) \\
\hline Centr. Afr. Rep. & 4,9 & (112) & 4,3 & (100) & 4,3 & (96) & 7,1 & (78) & 4,8 & (117) & 3,9 & (119) \\
\hline Romania & 4,9 & (112) & 4,0 & (102) & 5,6 & (65) & 2,7 & (122) & 6,4 & (78) & 5,5 & (78) \\
\hline Russia & 4,9 & (112) & 5,3 & (79) & 4,4 & (90) & 3,6 & (115) & 6,9 & (65) & 4,4 & (112) \\
\hline Syria & 4,9 & (112) & 3,7 & (109) & 5,1 & (75) & 6,6 & (93) & 6,1 & (89) & 2,7 & (123) \\
\hline Malawi & 4,7 & (116) & 3,9 & (106) & 5,5 & (66) & 2,8 & (120) & 5,9 & (100) & 5,1 & (99) \\
\hline Ukraine & 4,7 & (116) & 3,4 & (118) & 4,8 & (79) & 3,5 & (117) & 7,0 & (63) & 4,6 & (110) \\
\hline Congo, Rep. Of & 4,4 & (118) & 3,5 & (113) & 2,4 & (122) & 4,3 & (113) & 6,8 & (72) & 4,8 & (105) \\
\hline Guinea-Bissau & 4,4 & (118) & 4,6 & (91) & 3,0 & (119) & 3,3 & (118) & 5,9 & (95) & 5,1 & (97) \\
\hline Algeria & 4,3 & (120) & 3,9 & (107) & 2,9 & (120) & 5,8 & (109) & 5,6 & (106) & 3,2 & (122) \\
\hline Zimbabwe & 4,3 & (120) & 4,7 & (90) & 5,0 & (76) & 2,8 & (121) & 3,7 & (121) & 5,4 & (90) \\
\hline Myanmar & 3,6 & (122) & 3,5 & (114) & 3,2 & (118) & 5,4 & (111) & 1,7 & (122) & 4,0 & (116) \\
\hline Congo, Dem. R & 3,5 & (123) & 4,9 & (86) & 2,4 & (121) & 1,3 & (123) & 4,9 & (116) & 4,0 & (117) \\
\hline
\end{tabular}

Source: Gwartney, James and Lawson, Robert (2004).

The economies most open to foreign trade were Hong Kong, Singapore, and Ireland. Two former Soviet bloc nations also rank fairly high in openness to trade, Estonia in $7^{\text {th }}$ place and Hungary in 14th. The least regulated countries - those at the top in Area 5 - were Hong Kong, United Kingdom, and the United States. 
The data on economic growth, GDP, population growth and investment rate was taken from the Penn World Tables version 6.1. The first consists on the average real ${ }^{1}$ GDP per capita rate of growth (constant prices: Chain series) for five-year periods from 1970-2000 to be compatible with the available economic freedom data. GDP is also real product per capita (Constant Prices: Chain series). Population growth consists on the average rate of growth for five-year periods from 1970-2000. Investment rate is the investment share in Real GDP \% in 1996 constant prices. Finally, the data on schooling is the gross enrollment ratio in secondary school, taken from the UNESCO Database.

Graph 1 show us that there is a positive association between Economic Freedom and GDP per capita, Investment/GDP ratio, economic growth and investment in human capital, with schooling as proxy. For instance, the countries on the top EFN index quintile present an average GDP per capita growth rate of 2,25\% for the period 1995 2000 , while the bottom quintile present a negative average growth rate of $-0,79 \%$. We can also observe that the GDP per capita (1996 US\$) of the top quintile is above US\$23.000, while the bottom quintile presents income per capita level merely over US\$3.000. It also indicates that economic freedom, besides fostering economic growth on its own, might also enhances economic progress through another channel, that is, by stimulating capital accumulation, leading to a higher steady-state level of output. Indeed, we can also observe that the average Investment/GDP ratio of the top quintile is above $22 \%$, while for the bottom quintile this ratio level is below $10 \%$. To investigate the existence of such a channel, we look at correlation between the twenty-one subcomponents of economic freedom and the investment/GDP ratio. The results are presented in Table 3 (appendix). With a three exceptions (government consumption, transfers and subsidies and marginal income tax rate), the estimated correlations are positive. In particular, the investment rate is shown to be high when the intellectual property is protected, there is no military interference in the rule of law, there is access to sound money, legal system is upright and taxes on international trade are low.

$1 \quad$ PPP converted. 
AVGROWTH: average growth rate of real GDP per capita in the last five years;

LINC2000: natural log of the 2000 value of real GDP per capita;

LINC70: natural log of the 1970 value of real GDP per capita;

AVINVRATE: average investment/GDP ratio in the last five years;

AVPOPGR: average population growth in the last five years;

SCHOOL: gross enrollment ratio in secondary school

GRAPH 1- ECONOMIC INDICATORS AND THE EFN INDEX - YEAR 2000

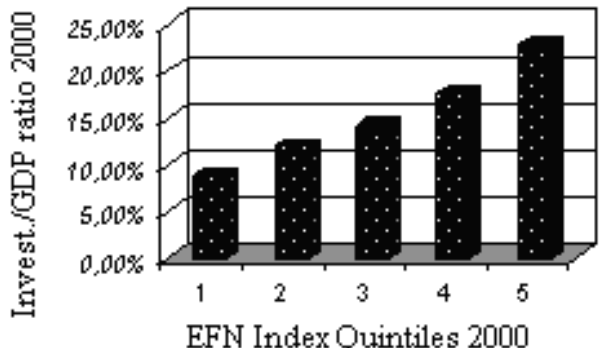

EFN Index Quintiles 2000

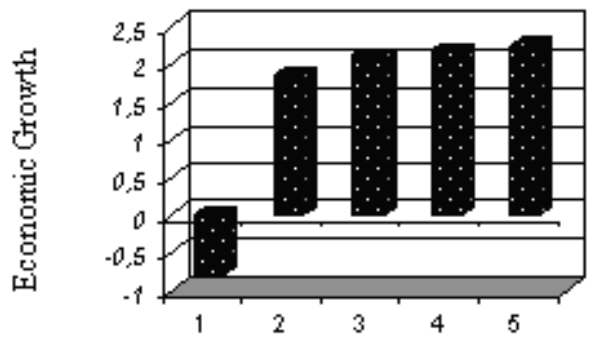

EFN Index Quintiles 2000

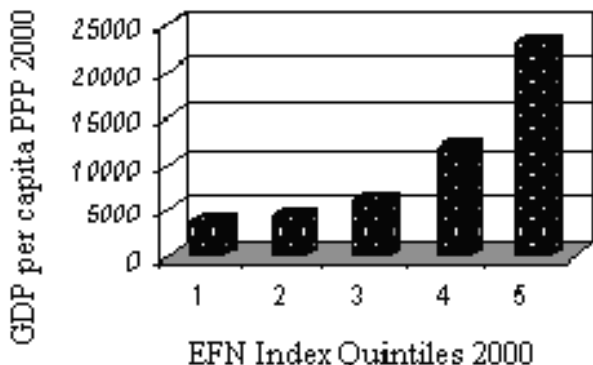

EFN Index Quintiles 2000

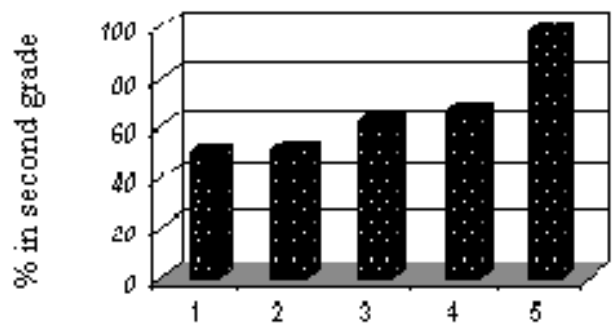

EFN Index Quintiles 2000

\section{TABLE 3 - CORRELATION BETWEEN ECONOMIC FREEDOM AND INVESTMENT RATE}

\begin{tabular}{cccccc}
\hline & avinvrate & \multicolumn{5}{c}{ avinvrate } & avinvrate \\
\hline area1a & -0.2312 & area2d & 0.4151 & area4b & 0.3629 \\
area1b & -0.1933 & area2e & 0.4837 & area4c & 0.0928 \\
area1c & 0.2959 & area3a & 0.3814 & area4d & 0.2430 \\
area1d & -0.1357 & area3b & 0.3729 & area4e & 0.2113 \\
area2a & 0.3394 & area3c & 0.3112 & area5a & 0.2668 \\
area2b & 0.2650 & area3d & 0.2832 & area5b & 0.0457 \\
area2c & 0.4436 & area4a & 0.4325 & area5c & 0.3108 \\
\hline
\end{tabular}




\section{GROWTH MODEL AND EMPIRICAL STRATEGY}

In this paper, we plan to understand which role economic freedom and its components play on growth. To study this matter, we present in this section a simple version of the standard neoclassical growth model with labor augmenting technical progress. This model will be the theoretical framework adopted here that will support and give solid grounds to our analysis.

Suppose the economy's aggregate production function is

$$
Y=F(K, A L)
$$

where $\mathrm{K}$ stands for total capital input, $\mathrm{L}$ for total labor input and $\mathrm{A}$ is a shift factor, where $\mathrm{A}>0$ and $\partial \mathrm{F} / \partial \mathrm{A}>0$. To simplify our exposition, we can actually think of $\mathrm{AL}$ as the amount of effective labor (i.e. labor units $\mathrm{L}$ multiplied by the technical shift factor A). So, output grows due not only to increases in capital and labor units ( $\mathrm{K}$ and $\mathrm{L}$ ), but also by increasing the effectiveness of each labor unit (A). Notice also that while the real rate of return on capital remains $r=F_{K}$, but the real wage is now $\mathrm{w}=\mathrm{A} .(\partial \cdot \partial \mathrm{F} . / \partial \mathrm{A} \cdot \mathrm{L})]=\mathrm{A} \cdot \mathrm{F}_{\mathrm{AL}}$.

Dividing (1) by $A L$ and rearranging terms, we have:

$$
y=A F(k)
$$

where $y=Y / A L$ and $k=K / A L$ are the output-effective labor ratio and capital-effective labor ratio, respectively.

Now, suppose that the physical labor units, $\mathrm{L}$, grow at the population growth rate $\mathrm{n}\left(\mathrm{g}_{\mathrm{L}}=\mathrm{n}\right)$, the technical shift factor A grows at the rate $\theta\left(\mathrm{g}_{\mathrm{A}}=\theta\right)$, $\mathrm{s}$ is the savings (and investment) rate, $\mathrm{d}$ is the depreciation rate of capital and $\mathrm{y}_{0}$ the initial level of output. The rate of growth of $\mathrm{y}(\hat{y})$ and the steady-state level of output $\left(y_{s s}\right)$ can be written as:

$$
\begin{aligned}
& \hat{y}=g\left(y_{o}, s, d, n ; A\right) \\
& y_{s s}=h(s, d, n ; A) \quad h_{s}
\end{aligned}
$$


with partial derivatives $g_{y_{0}}<0, g_{s}>0, g_{d}<0, g_{n}<0, g_{A}>0$ and $h_{s}>0, h_{d}<0$, $h_{n}<0, h_{A}>0$. We now hypothesize that economic freedom enhances the efficiency by which productive inputs are converted into output, that is, technology is an increasing function of Economic Freedom (EF): $A=A(E F)$, with $A^{\prime}>0$. Therefore, substituting that into (3a) and (4a), we find:

$$
\begin{aligned}
& \hat{y}=g\left(y_{o}, s, d, n ; E F\right) \\
& y_{s s}=h(s, d, n ; E F)
\end{aligned}
$$

Effective labor grows at rate $\mathrm{g}_{\mathrm{AL}}=\mathrm{g}_{\mathrm{A}}+\mathrm{g}_{\mathrm{L}}=\mathrm{n}+\theta$. For steady-state growth, capital must grow at the same rate as effective labor grows, in order to keep K/AL constant, then, in steady-state, $\mathrm{g}_{\mathrm{K}}=\mathrm{n}+\theta$.

While all the steady-state ratios - output per effective capita $\left(y_{s s}\right)$, consumption per effective capita $\left(c_{s s}\right)$ and capital per effective capita $\left(k_{s s}\right)$ - are constant, this is not informative of the welfare of the economy. It is real people - and not effective people - that receive the income and consume. In other words, to assess the welfare of the economy, we want to look at output and consumption per physical labor unit.

Now, the physical population $\mathrm{L}$ is only growing at the rate $\mathrm{n}$, but output and consumption are growing at rate $\mathrm{n}+\theta$. Consequently, output per person, $y_{s s}$ and $c_{s s}$ are growing at the steady rate $\theta$, the rate of technical progress. Thus, in the steady-state situation, output per person growth is determined by technological progress.

Equations (3b) and (4b) imply that, under the hypothesis we have made, economic freedom must have significantly positive effects on economic growth and the steady-state level of output when we control for the effects of initial income, investment rate and population growth rate. The functional form of these equations is another issue that is important to consider. It can to a certain extent be determined by using econometric tests, but should as far as possible be based on economic theory. It is not evident that the relation between economic freedom and growth is linear and the appropriate specification may differ among categories. For example, there could be diminishing returns in the relation between the size of government and growth. Moreover, a certain economic freedom category may only have a small effect on growth at low degrees of freedom, but a large effect at high degrees of freedom, or only have an effect if a critical level of freedom is reached. For example, increasing access to sound money from a very low level might not have an impact on the agents in the economy since their trust in the government, based on previous behavior, is still low. For simplicity, we estimate linear versions of (3b) and (4b), testing for several 
components of economic freedom, but we would like to stress that further research into the functional form of the model is needed.

\section{Empirical Analysis}

That economic freedom spurs economic growth by unleashing individual dynamism and improving productivity is hardly questionable. The matter here is to determine empirically which aspects of economic freedom mostly contribute to economic expansion and thereby to point out the issues which should have priority on the political effort to foster freedom. We believe that economic freedom is a very wide concept and we find that the synthesis of data on the index construction, although very useful, causes loss of relevant information. Thus, in this section we present a more specific kind of empirical analysis that treats more in detail this issue.

We estimate equations ( $3 b)$ and ( $4 b)$ assuming that the rates of saving and population growth are independent of country-specific factors shifting the production function (A), therefore, we can estimates both equations with pooled ordinary least squares (POLS). ${ }^{2}$

Table 4 (appendix) presents a number of linear specifications of equation (3b). We begin with specification (i) which ignores the economic freedom term and estimates a standard growth regression. The results from this specification are consistent with the implications of the neoclassical model. Because of conditional convergence, per capita income at the beginning of the period (1970) has a negative and statistically significant sign: ceteris paribus, countries that start poorer grow faster. The investment rate, human capital and population growth variables also have the expected signs (positive, positive and negative, respectively) and both are statistically significant.

In specifications (ii)-(xxiii) of Table 4, we successively include in the regression each of the twenty-one sub-components of the economic freedom index. Firstly, we find that the estimated coefficients and statistical significance of investment rate and income in 1970 are robust to the inclusion of the new variables and continue to be as predicted by the neoclassical model (population growth is somewhat less robust and becomes statistically insignificant in several cases). Second, the estimated coefficients of all the economic freedom variables have the expected sign (positive), although they are not all significantly different from zero. In particular, the empirical results enable us to identify fourteen components of economic freedom which have had statistically significant positive effects on growth, when one controls for the effects of initial income, the investment rate, human capital and the population growth rate.

2 This assumption was originally made by Mankiw, Romer and Weil (1992). 


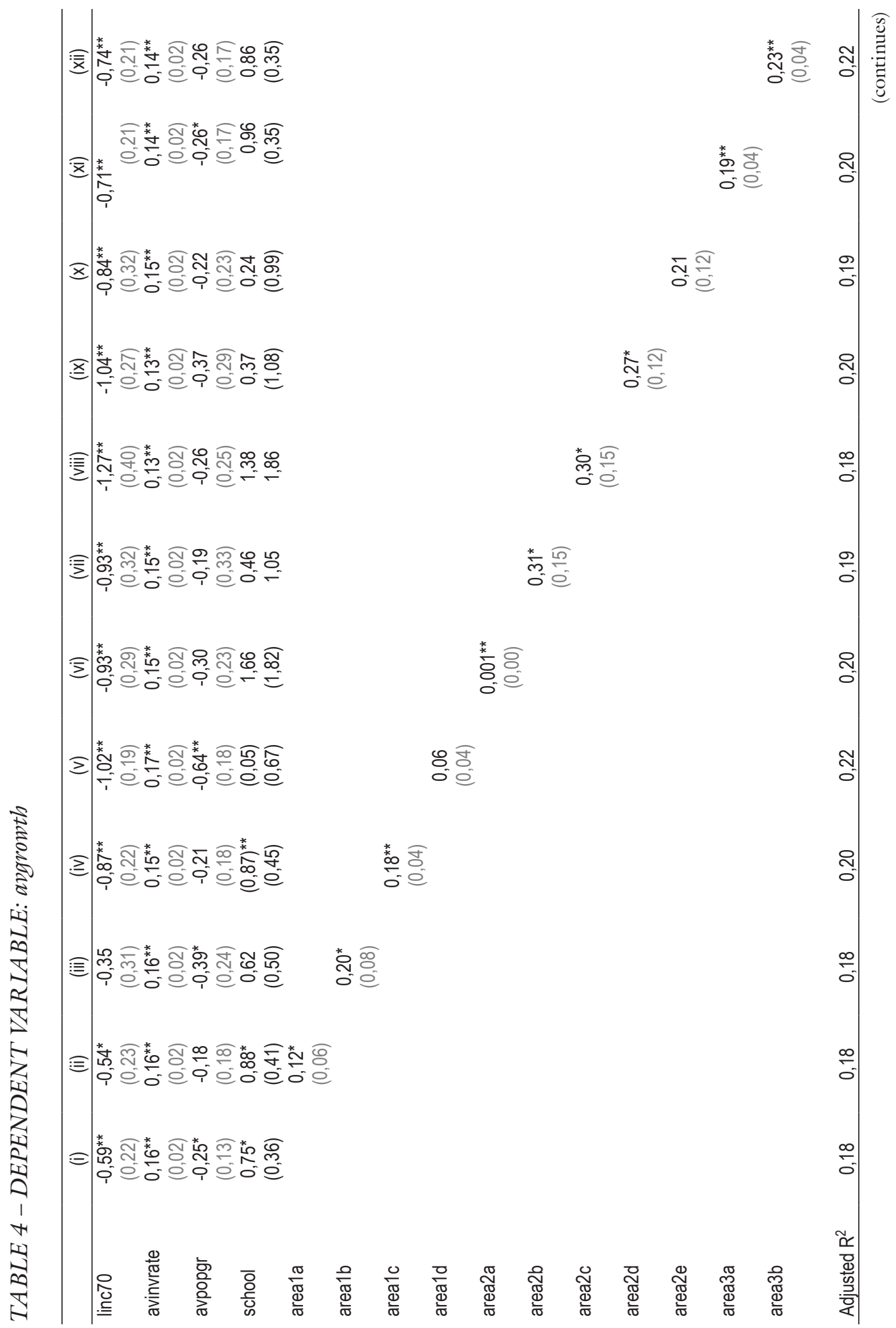




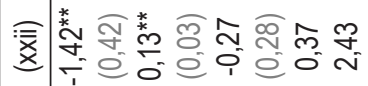

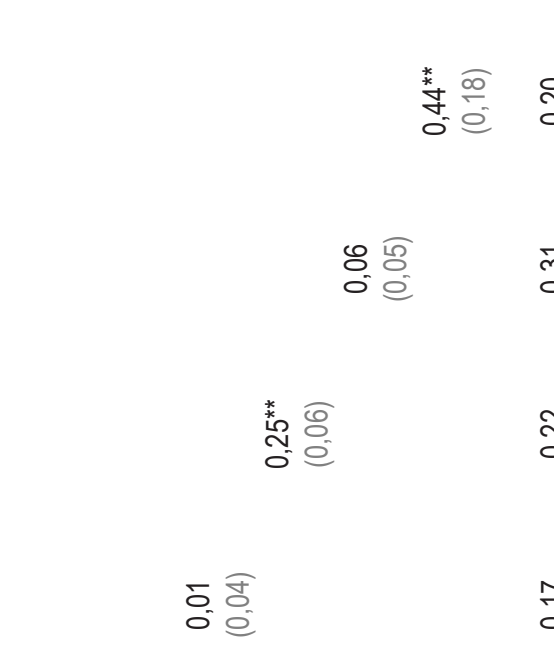

胥

$\begin{array}{ll}* & \\ 0 & 0 \\ 0 & 0 \\ 0 & 0\end{array}$

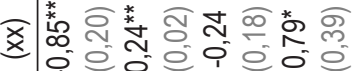

స్

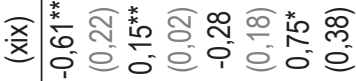

둥

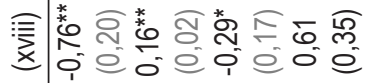

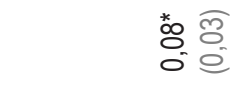

్ㅜㅇ

120
0
0

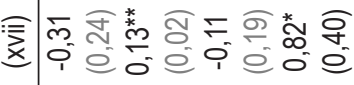

$\frac{0}{\circ}$

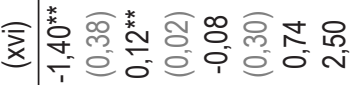

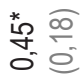

ำ

$\stackrel{\circ}{\circ}$

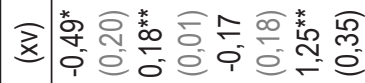

응응

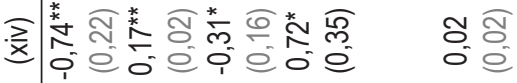

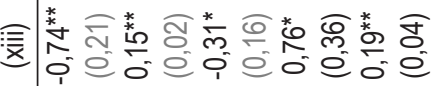


The finds in Table 4 show us that productivity (A) is enhanced when (i) government consumption is small compared to total consumption, (ii) transfer and subsidies are kept low, (iii) public sector responds for a low share of total investments, (iv) judiciary is independent, (v) courts are impartial and can be trusted, (vi) intellectual property is protected, (vii) there is no military interference in the political process, (viii) money growth is kept close to real GDP growth, (ix) low standard inflation variability in the last five years (x) low recent inflation levels, (xi) trade barriers are small and clear, (xii) official exchange rate is near black-market rate, (xiii) credit market is less regulated and (xiv) business and prices are less regulated. Surprisingly, marginal tax rate, integrity of legal system, freedom to own foreign currencies, taxes on international trade, size of international trade sector, foreign capital control and labor market regulations did not present statistically significant coefficients.

We obtain very similar results when we estimate a number of linear specifications of equation (4b), using the logarithm of the 2000 per capita income as a proxy for the steady state. Specification (i) once again ignores the economic freedom terms. Because of persistence, per capita income at the beginning of the period (1970) has a large positive effect on current income and it is statistically significant: ceteris paribus, countries that were rich in 1970 continue to be rich in 2000. The investment rate and population growth variables also have the expected signs (positive and negative, respectively) and both are (almost in every case) statistically significant.

As in Table 4, specifications (ii)-(xxiii) of Table 5 successively include in the regression each of the twenty one economic freedom variables. The estimated coefficients and statistical significance of the three first variables are even more robust to the inclusion of the economic freedom variables then for equation (4a). All specifications show very high adjusted goodness-to-fit $\mathrm{R}^{2}$ measure, never lower than 0,87 . Also, the estimated coefficients of all the economic freedom variables have the expected positive sign, and thirteen of them are significantly different from zero. We also note that the list of statistically significant economic freedom components is similar to the one produced by Table 4 . The difference appears basically in the coefficient of government consumption, transfer and subsidies, military interference in the rule of law and difference between official exchange rate and black-market rate. These coefficients cease to be statistically significant. On the other hand, the coefficient for labor market regulation becomes significant to explain steady-state income level. 


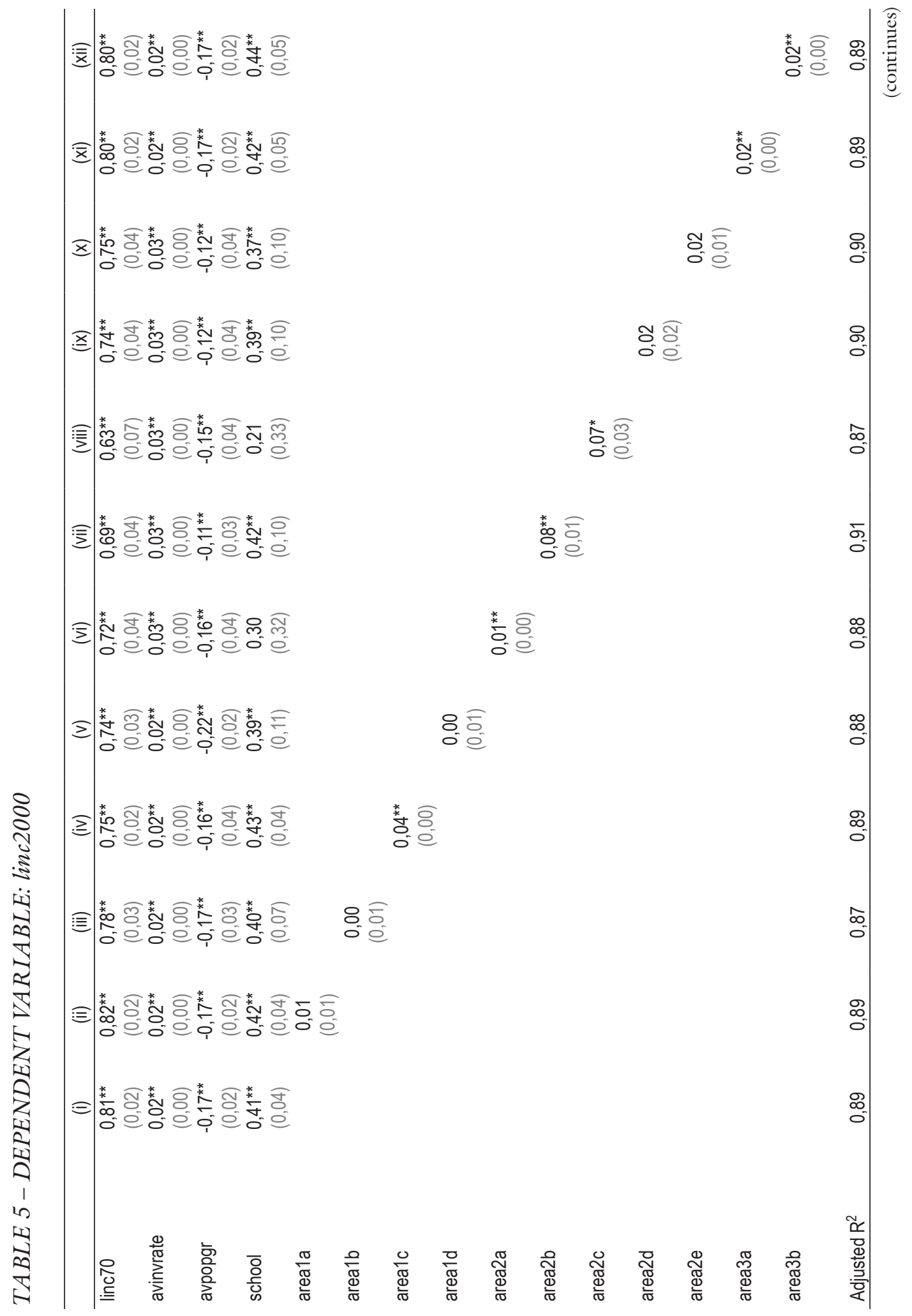




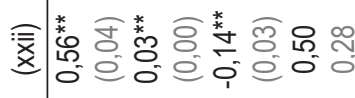

으응

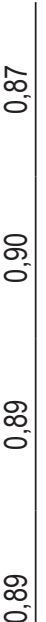

畓

$*$
*ิ
0
0

응

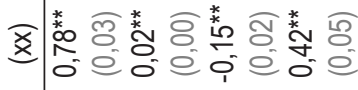

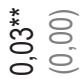

\&

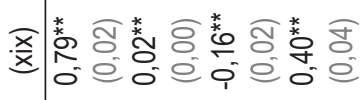

$*$
$\overline{0}$
0

$\begin{array}{ll}* & \\ \delta & 0 \\ 0 & 0\end{array}$

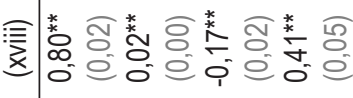

б흐

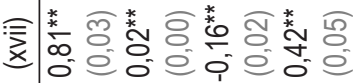

증

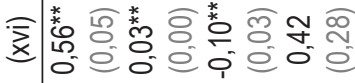

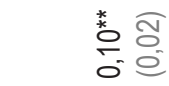

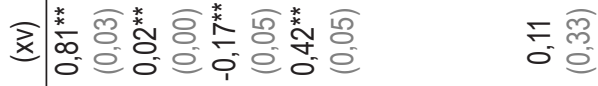

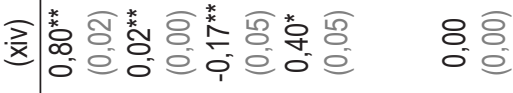

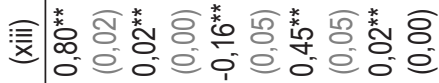

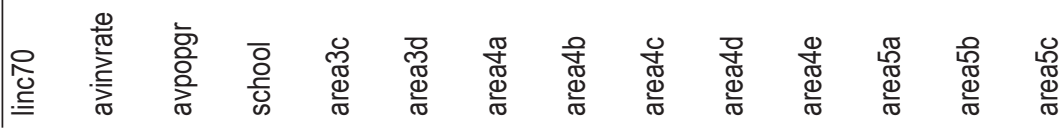




\section{Granger-Causality}

Clive Granger (1980) proposes the following definition of causality: a (time series) variable $A$ causes $B$, if the expected value of $B$ conditional on its own past history and the past history of $A$ does not equal the probability of $B$ conditional on its own past history alone. More formally, we say that A Granger causes B if

$$
\mathrm{E}\left(A_{t} / I_{t-1}\right) \neq \mathrm{E}\left(A_{t} / J_{t-1}\right)
$$

where $\mathrm{I}_{\mathrm{t}-1}$ contains past information on $\mathrm{A}$ and $\mathrm{B}$, and $\mathrm{J}_{\mathrm{t}-1}$ contains only information on past A. This definition, in the econometric literature referred to as Granger-causality, has been extensively applied in econometrics because it has the advantage, as it has been emphasized by Granger himself, to be operational.

We now perform tests for Granger-causal relationships between economic freedom and growth using several different equations. The first group of equations are used to test for unilateral Granger causality of the level of economic growth, that is, they allow for tests to determine if economic freedom Granger-causes economic growth. Those relationships are examined in time $t$ for country $i$ using growth (average real GDP per capita rate of growth) and $E F^{A}$ for $\mathrm{A}=1,2, \ldots, 21$, representing the 21 sub-areas that form the EFN Index, as a measure of the different components of economic freedom:

$$
\operatorname{growth}_{t, i}=\alpha_{0, i}+\sum_{k=1}^{K} \beta_{k} \text { growth }_{t-k, i}+v_{t, i}
$$

(2)-(22) growth $_{t, i}=\alpha_{A, i}+\sum_{k=1}^{K} \beta_{k}$ growth $_{t-k, i}+\sum_{j=1}^{J} \theta_{A j} E F_{t-j, i}^{A}+\bigcup_{t, i} \quad$ for $\mathrm{A} \in[1,21]$

where $v_{\mathrm{t}}$ is assumed to be a white noise term and growth $\mathrm{t}_{\mathrm{t}-\mathrm{k}}$ and $\mathrm{EF}_{\mathrm{t} \cdot \mathrm{j}}^{\mathrm{A}}$ are lagged values. The number of lagged values ( $\mathrm{K}$ and $\mathrm{J}$ ) is chosen by the investigator. To conduct the Granger-causality test, all the regression equations above are estimated and followed by an $F$-test in order to test the null hypothesis that $\theta_{\mathrm{Aj}}=0$ for $\mathrm{J}=1,2, \ldots \mathrm{J}$. A rejection of the null hypothesis implies that the relevant measure of economic freedom $\left(\mathrm{EF}^{\mathrm{A}}\right)$ Granger-causes economic growth.

Equation ( 1 ) is used to control for the information contained in lagged values of the dependent variable while equations (2) - (22), which are extensions of equation (1), also include the information contained in lagged values of the sub-areas of economic freedom. In these equations, $\theta_{\mathrm{Aj}}$ for $\mathrm{j}=1,2, \ldots, \mathrm{J}$ are interpreted as the absolute change in the rate of growth that results from a previous one-unit change in the measures 
of economic freedom. If equation (2), for example, makes a significant contribution to the explanation of growth over and above equation (1), this would mean that prior levels of $\mathrm{EF}^{\mathrm{l}}$ are significantly related to the current level of economic growth, leading us to the conclusion that $\mathrm{EF}^{1}$ Granger-causes growth (same applies for all other equations).

Symmetrically, the next group of equations are used to test for unilateral Granger causality of economic freedom, that is, they allow for tests to determine if economic growth Granger-causes economic freedom.

$$
E F_{t, i}^{A}=\lambda_{A, i}+\sum_{m=1}^{M} \gamma_{m} E F_{t-k, i}^{A}+v_{t, i}
$$

(44)-(64) $E F_{t, i}^{A}=\lambda_{21+A, i}+\sum_{m=1}^{M} \gamma_{m} E F_{t-k, i}^{A}+\sum_{n=1}^{N} \delta_{A n}$ growth $_{t-n, i}+\mho_{t, i}$ for $\mathrm{A} \in[1,21]$

Finally, we shall also analyze and compare the results from the unilateral tests shown in equations (l) through (64). For example, if $\mathrm{EF}^{\mathrm{l}}$ is shown to Granger-cause growth using results from the first two equations and growth is shown to Granger-cause EF using the information provided in equations (23) and (44), the conclusion would be that the two are bilaterally related or jointly determined. Similar information can also be drawn for other possible bilateral relationships shown in the specified equations.

Dummy variables for all countries were included when estimating each of the specified regression equations to help control for country-specific information. This is necessary because our data set includes a limited number of time-series observations from a large number of countries and when these are pooled to capture and exploit the time-series properties of all countries together in an effort to explore for causal relationships, there is a risk that cross- country differences may be so significant as to overwhelm any time-series information that is available in the data, biasing the results and providing an inaccurate picture of any "true" relationship that may exist among the variables being studied. ${ }^{3}$ These dummies do not have their parameter estimates shown in the tables so that attention can be focused on the parameters that are pertinent for testing.

The ordinary least-squares parameter estimates for equations to test if Granger causality does run unilaterally from economic freedom to growth are shown in Tables 6. A priori, it is expected that, if a significant relationship is found to exist among any of the variables being studied, it should be positive.

3 See Farr, Lord and Wolfenbarger (1998). 


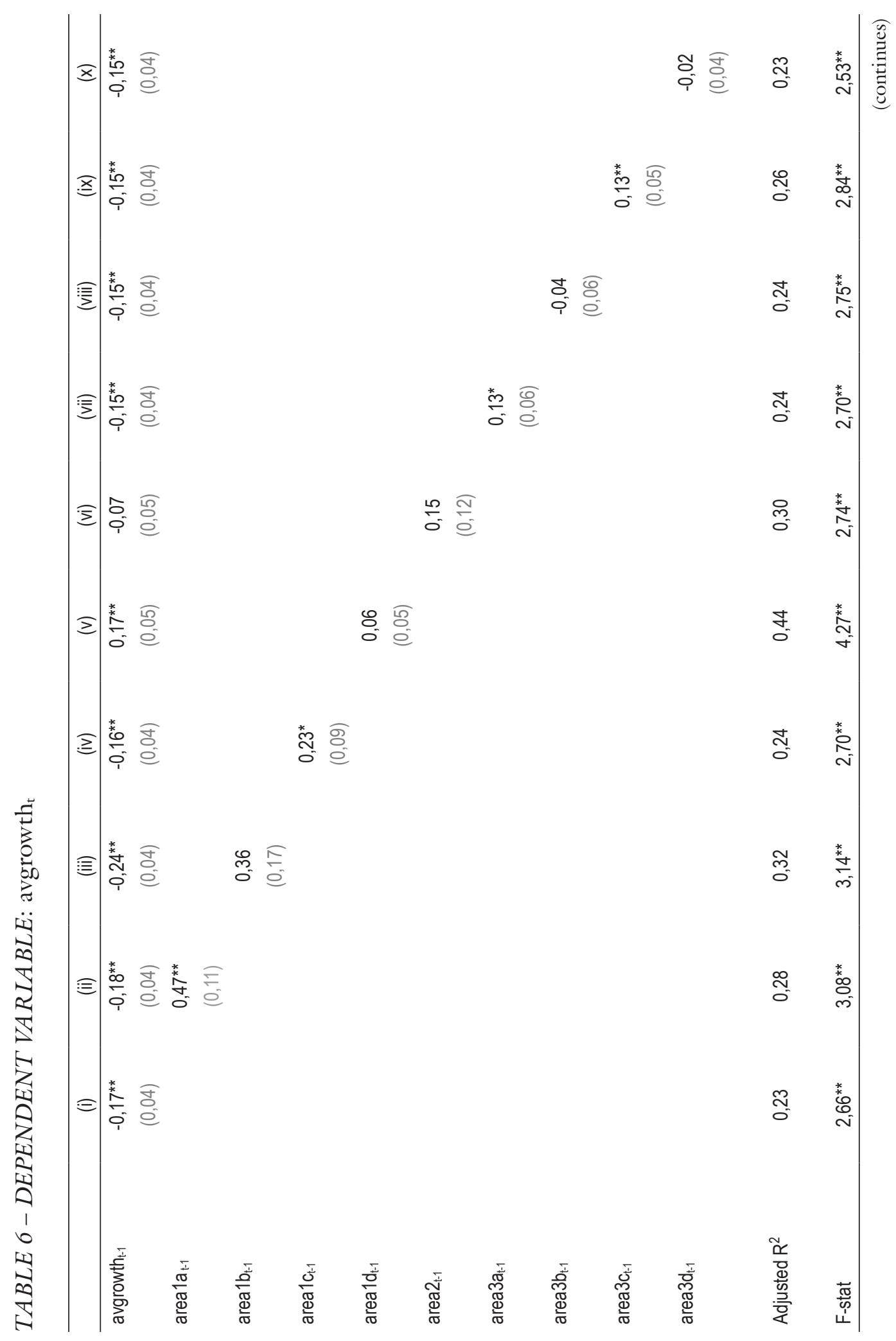




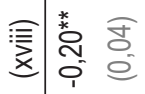

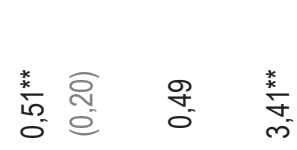

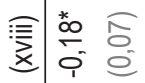

$\begin{array}{ll}8 & 0 \\ 0 & 0\end{array}$

잉

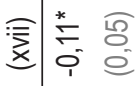

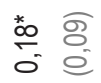

mi

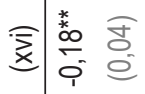

б.

ิํำ

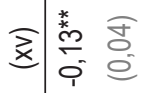

ஜ 요

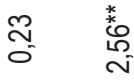

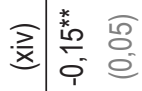

응 응

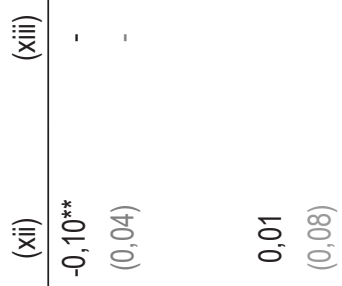

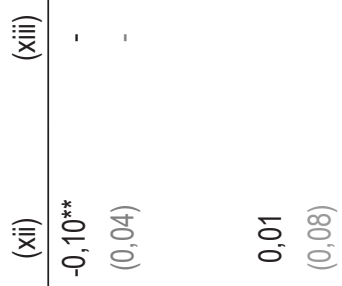

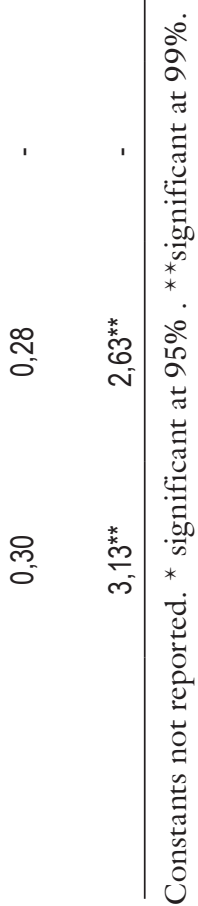

ָㅡㄴ

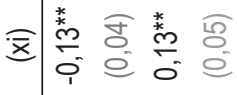


We begin with specification (i) which ignores all economic freedom terms and estimates a simple regression of growth on its lagged value growth ghe $_{t-1}$ The results from this specification are consistent with the implications of the neoclassical model. Because of conditional convergence, current economic growth is has a negative and statistically significant relationship with lagged growth. When we also include a twice lagged variable of economic growth, both coefficients appear to be negative and significant (results not shown).

In specifications (ii)-(xix) of Table 6, we successively include in the regression almost every one of the twenty-one sub-components of the economic freedom index (some of them have not been tested due to lack of data availability). Firstly, we find that the estimated coefficients and statistical significance of growth $_{t-1}$ are robust to the inclusion of the new variables. Second, the estimated coefficients that are statistically significant have the expected sign (positive). In particular, the empirical results enable us to identify which components of economic freedom past values have had statistically significant positive effects on current rate of growth, when one controls for the lagged growth. Firstly, all F-statistics are statistically significant at 99 percent level. On the other hand, the t-statistics are only significant for government consumption (areala), public investment (arealc), growth of money supply (area3a), recent inflation (area3c), freedom to trade internationally (area4), regulation on credit market (area5a) and on business market (area5c). That is, the rate of economic growth should be higher in the future in countries where, ceteris paribus, government consumption expenditure is kept low, investment is primarily made by private agents, growth of money supply is kept near to the growth of per capita income, recent inflation is low, people are free to trade with the rest of the world and credit and business markets are deregulated.

All sub-areas under 2 - Legal Structure and Security of Property Rights have not been tested due to missing data (same applies for area $4 \mathrm{~b}$ ). We do test the coefficient for area 2 as a whole, but it fails to be significant at 95 percent. This result could come to us as a shock as we would suppose that a sound legal structure and good protection of property rights should spur growth on a large scale. Unfortunately, given the methodology used in this study, the limited amount of available data is not sufficient to adequately explore for that possibility. And even if there was more data available, the difficulty to measure accurately legal institutional framework would represent an extra obstacle for the objective measurement of that area of economic freedom.

Although not a single sub-area of area 4 - Freedom to trade internationally shows significant coefficients, the coefficient for area 4 itself is positive and highly significant, suggesting that freedom to trade internationally does indeed enhance economic 
growth (this fact does not seem to be troublesome because all items under area 4 seem to be related and do not appear to influence growth on different directions).

On Table 7, we find the ordinary least-squares parameter estimates for equations to test if economic growth Granger-causes economic freedom. For every relevant subarea of the EFN index separately (again, sub-areas under area 2 and $5 b$ have not been tested), we begin with specification (i) which ignores all economic growth terms and estimates a simple regression of freedom on its lagged value area $a_{t-1}$. All coefficients but two are statistically significant. Then, in specification (ii) we include a lagged variable of economic growth $\left(\right.$ growth $\left._{\mathrm{t}-1}\right)$ to check if we can improve the predictability of current level of economic freedom when including information based on past growth. Results show that, after controlling for past economic freedom, past growth is positively correlated with Standard inflation variability in the last five years and negatively correlated with Transfers and subsidies as a percentage of GDP, Top marginal tax rate, Freedom to trade internationally and taxes on international trade.

These results indicate that economic growth, although enhanced by freer economic environments, act as an obstacle for further expansions of economic freedom. Economic expansion seems to Granger-cause a more than proportional raise on government transfers and subsidies and on tax rate. We do not have a clear insight on why this should be, but we do suspect that it is related to the idea that governments might tend to try to correct the bad side of a perhaps unfair economic growth by transferring resources to low-income fractions of the population that are not enjoying the benefits from this growth. This could also explain higher top marginal tax rate, because governments worried about equality would try to take larger portions of income from the wealthy to raise fund for higher transfers and subsidies. This could be particular true for social-democrat nations. The results also tell us that past economic growth is related to current loss of freedom to trade internationally. This finding goes against our intuition that fast-growing countries would seek to open their economy trying to have access to larger markets, because of their also growing competitiveness and productivity. On the other hand, we should consider the possibility that economic growth strengthens the power of some pressure groups, and these groups could on their turn exercise their lobby power in order to annulate international competition.

Finally, the only sub-area of economic freedom that appears to have bilateral relationship with growth is the freedom to trade internationally. While more open economies appear to show higher rates of economic expansion, growth tends to minimize this effect by negatively influencing the extent to which countries are free to trade with others. 


\section{TABLE 7 - PAST GROWTH AND CURRENT ECONOMIC FREEDOM}

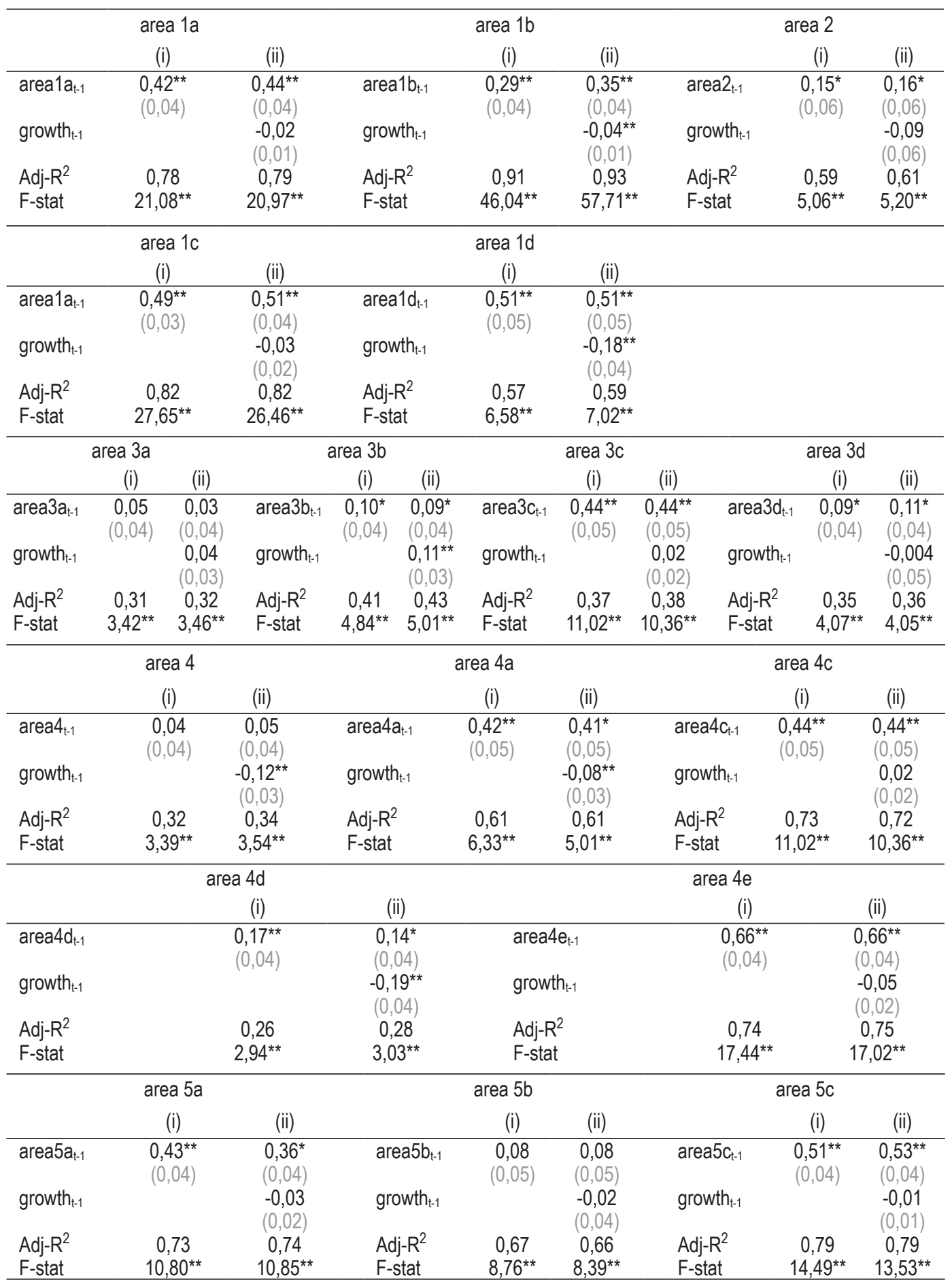




\section{CONCLUSION AND SUGGESTIONS ON POLICY}

The findings presented here indicate that adoption and maintenance of institutions and policies consistent with economic freedom are vitally important for the achievement of prosperity. Countries with more economic freedom attract more investment and achieve greater productivity from their resources. As a result, they grow more rapidly and achieve higher income levels. In contrast, countries stagnate when their institutions stifle trade and erode the incentive to engage in productive activities. Provision of institutions supportive of economic freedom is the key to the growth process. Countries with low initial levels of income, in particular, are able to grow rapidly and move up the income ladder when their policies are supportive of economic freedom.

The results reported above are very supportive of the proposition that economic freedom enhances growth both via increasing total factor productivity and via enhancing capital accumulation. We expect this finding to be neither surprising nor controversial. What we consider to be the primary contribution of this paper to the literature is the identification of the sub-components of economic freedom which are shown to be statistically significantly correlated with growth. These are (i) small government consumption, (ii) low transfer and subsidies, (iii) public sector responding for a low share of total investments, (iv) independent judiciary, (v) impartial and reliable courts, (vi) protected intellectual property, (vii) no military interference in the political process, (viii) money growth kept close to real GDP growth, (ix) low standard inflation variability in the last five years (x) low recent inflation levels, (xi) small and clear trade barriers, (xii) official exchange rate near black-market rate, (xiii) less regulated credit market and (xiv) less regulated business markets.

We believe these findings are more useful than results which rely on aggregate or composite indices of economic freedom. While earlier research has shown economic freedom "as a whole" to be statistically significantly correlated with growth, our results show that this may be due to the effects of only some of the underlying components of economic freedom. An additional benefit of our analysis is that it can be used to offer more specific policy suggestions. Instead of much of the existing literature's vague recommendation of "enhancing economic freedom," our results have identified particular elements, cultivation of which is likely to promote capital accumulation and growth.

Still, however, a number of unresolved questions remain. For example, we are surprised that some of the variables (such as the marginal tax rate and foreign capital control) were not statistically significant. We suspect that this may reflect lack of 
identification power rather than the absence of an economic effect, but future research is necessary to address these questions more appropriately.

\section{BIBLIOGRAPHY}

Ayal, Eliezer B.; Karras Georgios. Components of economic freedom and growth: an empirical study. Journal of Developing Areas 32, p. 327-38, Spring 1998.

Berggren, N. Economic freedom and equality: friends or foes? Public Choice, v. 100, n. 3/4, p. 203-23, 1999.

Easton, Stephen T; Walker, Michael A. Income, growth, and economic freedom. American Economic Review, American Economic Association, v. 87, n. 2, p. 328-32, May 1997.

Esposto, A.; Zaleski, P. Economic freedom and the quality of life. Constitutional Political Economy, 10, p. 185-197, 1999.

Farr, W.; Lord, R.; Wolfewnbarger, J. Economic freedom, political freedom, and economic well-being: a causality analysis. The Cato Journal, v. 18, n. 2, Fall 1998.

Friedman, Milton. Economic freedom, human freedom, political freedom. Speech delivered at The Smith Center for Private Enterprise Studies, Hayward: California State University, 1991.

Gwartney, James; Lawson, Robert. Economic freedom of the world: 2004 annual report. Vancouver: The Fraser Institute, 2004. Data retrieved from www.freetheworld. com.

Gwartney, James; Lawson, Robert; Holcombe, Randall. Economic freedom and the environment for economic growth. Journal of Institutional and Theoretical Economics, v. 155, n. 4, p. 1-21, 1999.

Haan, Jakob de; Siermann, Clemens L. J. Further evidence on the relationship between economic freedom and economic growth. Public Choice, v. 95, n. 3-4, p. 363-80, June 1998.

Heston, Alan; Summers, Robert; Aten, Bettina. Penn World Table Version 6.1. Center for International Comparisons at the University of Pennsylvania (CICUP), October 2002.

Mankiw, N. Gregory; Romer, David; Weil, David N. A contribution to the empirics of economic growth. NBER Working papers series n. 3541, 1992.

Norton, Seth W. Poverty, property rights, and human well-being: a cross-national study. Cato Journal, v. 18, n. 2, p. 233-45, Fall 1998.

Smith, Adam. [1776] An inquiry into the nature and causes of the wealth of nations. University Of Chicago Press, 1977. Reprinted. 
Solow, Robert M. A contribution to the theory of economic growth. Quarterly Journal of Economics, p. 65-94, February 1956.

$\mathrm{Wu}$, Wenbo; Davis, Otto A. Economic freedom and political freedom. Forthcoming in Encyclopedia of Public Choice, 2005.

.The two freedoms, economic growth and development: an empirical study. Public Choice, v. 100, n. 1/2, p. 39-64, July 1999. 